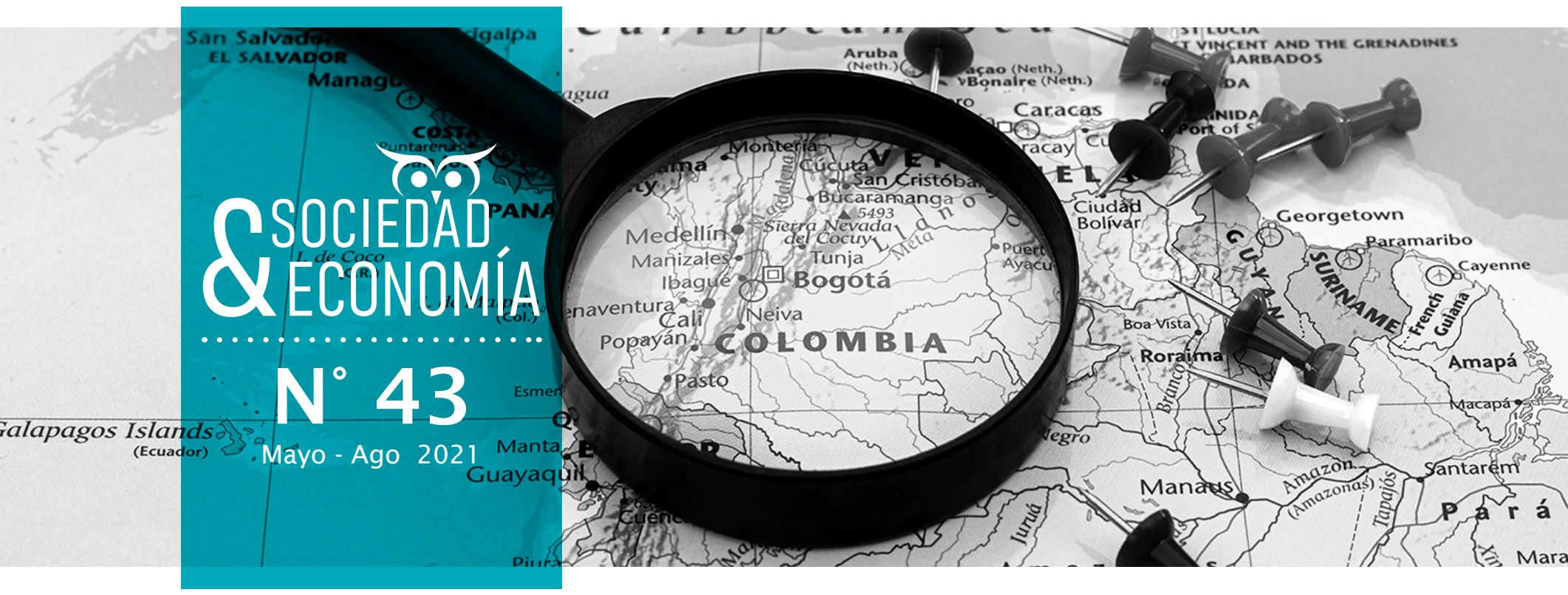

\title{
De lo simple a lo complejo: tres décadas del análisis de convergencia regional
}

\author{
From the Simple to the Complex: Three Decades of the \\ Analysis of Regional Convergence
}

\section{Angela Milena Rojas-Rivera'}

Universidad de Antioquia, Medellín, Colombia.

실. amilena.rojas@udea.edu.co

(D) https://orcid.org/0000-0003-3675-240X

\section{Juan Camilo Rengifo-López ${ }^{2}$}

Universidad Nacional, Medellín, Colombia.

@ jurengifol@unal.edu.co

(1) https://orcid.org/0000-0002-4920-5707
Recibido: 04-06-2020

Aceptado: 05-11-2020 Publicado: 01-05-2021

1 Doctora en Economía.

2 Economista. 


\title{
Resumen
}

El objetivo de este artículo es brindar una visión de conjunto de la investigación sobre la hipótesis de convergencia neoclásica en Colombia. Con este fin, se realiza una revisión de la literatura internacional y nacional, donde la primera contextualiza a la segunda. Asimismo, se destaca la conexión de los hallazgos con la política de desarrollo regional. Se encuentra que, en los estudios sobre convergencia regional colombiana, el análisis tradicional ha sido el método predominante mientras que el análisis de dinámica distributiva y efectos espaciales ha tenido menor incidencia. Igualmente, se verifica una lenta entrada de las teorías del crecimiento endógeno y la Nueva Geografía Económica. Ante la ausencia de convergencia regional en ingresos, evidenciada al menos desde la década de 1990, y la polarización en su distribución, las recomendaciones de política se orientan al activismo a través del gasto y la inversión pública, y al diseño de intervenciones espacialmente diferenciadas.

Palabras clave: hipótesis de convergencia; crecimiento económico regional; desequilibrio regional; políticas de desarrollo regional; desarrollo social.

Clasificación JEL: R11, R12, O18.

\begin{abstract}
The objective of this article is to provide an overview of the research on the neoclassical convergence hypothesis in Colombia. To this end, a survey of the international and national literature is carried out, in which the former contextualizes the latter. It also highlights the connection of the findings with regional development policies. It is found that in the studies on Colombian regional convergence traditional analysis has been the predominant method while the analysis of distributive dynamics and spatial effects has had the lowest incidence. Similarly, a slow entry of the endogenous growth theories and New Economic Geography is verified. In the absence of regional income convergence, evidenced at least since the 1990s and polarization in their distribution, policy recommendations are oriented towards activism through public spending and investment as well as the design of spatially differentiated interventions.
\end{abstract}

Keywords: Convergence hypothesis; regional economic growth; regional imbalance; regional development policies; social development.

JEL Classification: R11, R12, 018.

\section{Finaciación}

Este proyecto recibió el apoyo financiero del Departamento de Economía de la Universidad de Antioquia.

\section{Conflicto de interés}

Los autores declaran no tener ningún conflicto de interés en la publicación de este artículo.

Este trabajo está bajo la licencia Atribución-No-Comercial 4.0 Internacional

¿Cómo citar este artículo?

Rojas-Rivera, A. M. y Rengifo-López, J. C. (2021). De lo simple a lo complejo: tres décadas del análisis de convergencia regional. Sociedad y economía, (43), e1049951. https://doi. org/110.25100/sye.v0i43.9951 


\section{Introducción}

¿Cómo han estudiado los economistas las disparidades económicas entre regiones subnacionales en diversos países y, en particular, en Colombia? ¿Qué han encontrado? ¿Qué debates o consensos existen? Estas son las preguntas en torno a las cuales gira esta revisión de literatura, motivadas por la necesidad de construir una guía para navegar en una amplia literatura dedicada a explicar las diferencias y la dinámica de los niveles de riqueza y desarrollo socioeconómico a nivel subnacional. El gran interés en este tema refleja su importancia en la agenda de desarrollo, especialmente cuando, en las últimas décadas, la región subnacional se plantea como protagonista de la innovación productiva, la gobernanza local e incluso la globalización. De aquí que un interrogante fundamental se añada a los anteriores: ¿qué recomendaciones para la política pública en el país se derivan de estos hallazgos?

Para abordar estas preguntas, este artículo se concentra en la literatura que parte de la noción de convergencia en ingresos, cuya base teórica es neoclásica e incluye dos de sus extensiones. A saber, la teoría del crecimiento endógeno y la nueva geografía económica. Sin desconocer la existencia y aporte de otros enfoques teóricos en economía (con autores como Myrdal o Kaldor) o disciplinares (como la geografía económica o la historia económica), para comprender las brechas regionales, esta delimitación permite abordar estudios con una base teórica común que, además, constituyen el grueso, aunque no la totalidad, de esta literatura. Así mismo incluye una noción derivada, la convergencia social, la cual se aplica a indicadores sociales, generalmente en salud y educación, como variables próximas y medidas no monetarias de la calidad de vida.

Los objetivos de esta revisión son tres: en primer lugar, exponer brevemente los elementos teóricos y los métodos aplicados a la convergencia regional a nivel internacional, basándonos en las revisiones de literatura más destacadas (Fingleton y LópezBazo, 2006; Magrini, 2004; Rey y Janikas, 2005; Roberts y Setterfield, 2010). En segundo lugar, evidenciar hasta qué punto tales desarrollos a nivel internacional han influenciado los estudios sobre la convergencia regional colombiana. Los investigadores colombianos han sido bastante activos en este tema desde la década de 1990 hasta la actualidad, motivados en gran parte por la descentralización y la profundización de las brechas económicas regionales. En tercer lugar, esta revisión se propone identificar las grandes recomendaciones derivadas de esta literatura colombiana para la política de desarrollo regional. El propósito general está en brindar una visión de conjunto de la investigación colombiana sobre las brechas económicas regionales, contextualizada en el ámbito internacional y en conexión con la perspectiva sobre la política pública. Este ejercicio principalmente ofrece una carta de navegación sobre lo logrado y, en segunda instancia, señala a grandes rasgos algunas de las rutas disponibles para continuar la investigación y orientar el debate público. Una revisión de literatura semejante aún no se encuentra publicada.

El artículo contiene cinco secciones además de esta introducción. La segunda expone los fundamentos teóricos y la evolución de los métodos aplicados al estudio de la convergencia regional. La tercera presenta las investigaciones más representativas utilizadas en el caso colombiano e identifica sus métodos y principales resultados. La cuarta ofrece una valoración global del avance de la literatura internacional y nacional; sintetiza los resultados sobre la existencia de la convergencia en ingresos y social en el país, e identifica las implicaciones de política más destacadas. Finalmente, se encuentran las conclusiones.

\section{Teorías y métodos para el estudio de la convergencia regional}

A continuación, teniendo como enfoque la teoría neoclásica, se explican los métodos para el estudio de la convergencia regional existentes en la literatura.

\subsection{La teoría neoclásica del crecimiento económico}

El modelo de Solow (1956) y Swan (1956) establece el mecanismo teórico por el cual la tasa de crecimiento de una economía evoluciona a través del tiempo. Partiendo de la función de producción neoclásica y su maximización in- 
tertemporal sujeta a una restricción presupuestal, se deriva la ecuación dinámica de acumulación de capital. El modelo establece que entre más bajo sea el nivel inicial de capital per cápita, más rápida será su tasa de crecimiento y, por tanto, la tasa de crecimiento del producto per cápita. Si dos economías tienen estructuras similares, el modelo implica que aquella economía con menor capital per cápita inicial crecerá más rápido hasta alcanzar el nivel de ingreso per cápita de la economía con la mayor tasa capital-trabajo. De esta manera, el ingreso per cápita de las economías convergerá hacia el mismo nivel de equilibrio de largo plazo (o estado estacionario), cerrando la brecha inicial. A la existencia de este mecanismo nos referiremos como la hipótesis de convergencia, la cual tiene dos versiones: absoluta y condicional.

El marco para abordar la convergencia entre países será trasladado al estudio de la convergencia regional subnacional, a pesar de que los conceptos de nación y región están lejos de ser intercambiables. Precisamente, los efectos provenientes de la movilidad de factores y bienes entre regiones, y las externalidades espaciales estarán en el centro de la discusión, como se verá.

Teniendo como punto de partida los métodos aplicados, se distinguen tres grandes grupos: el análisis tradicional, la dinámica distributiva y el análisis espacial. El primero corresponde a un análisis confirmatorio al realizar la verificación empírica de la hipótesis neoclásica de convergencia. El segundo es un análisis exploratorio en tanto se concentra en determinar las propiedades estadísticas de las variables bajo estudio (Rey y Janikas, 2005). Finalmente, el análisis espacial presenta aplicaciones de tipo confirmatorio y exploratorio.

\subsubsection{Análisis tradicional (AT)}

El método más simple para verificar la predicción neoclásica de convergencia, en una regresión de mínimos cuadrados en corte transversal, utiliza una ecuación que describe la dinámica del ingreso per cápita y que corresponde a una aproximación lineal de la ecuación de acumulación de capital alrededor del estado estacionario. Allí la variable dependiente es la tasa de crecimiento del ingreso per cápita entre el período 0 y t, y la variable independiente es el logaritmo del nivel de ingreso per cápita en el punto inicial:

$(\mathrm{t}=0): \log [y(t) / y(0)]=a+b \log y(0)$.

La velocidad de convergencia hacia el estado estacionario, designada con el parámetro $b$, toma valores negativos, ya que se relaciona inversamente con la tasa capital-trabajo. A menor capital relativo más alta será la tasa de crecimiento y, por tanto, más rápida será la convergencia. Esta convergencia es Ilamada absoluta, ya que asume que las economías comparten los mismos parámetros $y$, por tanto, se aproximan al mismo estado estacionario de acuerdo con Barro y Sala-i-Martin (2003).

No obstante, dado que la tasa de ahorro y el avance tecnológico pueden diferir entre países, aparece la noción de convergencia relativa o convergencia condicional, en donde la velocidad de convergencia $(\beta)$ está condicionada al ingreso per cápita de su propio estado estacionario, la tasa de crecimiento exógena de la tecnología y el nivel inicial de tecnología. La ecuación lineal reducida ahora se convierte en:

$(1 / t) \log [y(t) / y(0)]=c-\left(1-e^{-\beta t}\right) / t * \log y(0)+u(t)$, donde $u(t)$ es un término aleatorio y la constante resume los parámetros no observables. Para verificar econométricamente esta ecuación, el conjunto de datos debe estar condicionado a los diversos estados estacionarios. Una forma de lograr esto es seleccionar un grupo homogéneo de países que compartan tecnologías y marcos institucionales similares (ej. Países de la Unión Europea). Si los países son heterogéneos, entonces se introducen variables explicativas adicionales como proxy de los diferentes estados estacionarios.

Cuando el análisis se aplica a regiones al interior de un país o bloques de países económicamente integrados, el supuesto de independencia entre unidades de análisis no se cumple y el cálculo del parámetro $\beta$, usando el estimador de mínimos cuadrados, resulta sesgado. Una primera respuesta a este problema es descomponer el error en dos partes, $u(t)=\varphi s(t)+v(t)$, de tal forma que el primer término represente el error agregado y, el segundo, el error aleatorio, asumiendo que el término $\varphi$ se distribuye independiente de $v(t)$, y que $\operatorname{cov}[\log y(0), \varphi]=0$. 
Según Magrini (2004), a partir de los trabajos de Baumol (1986) y Barro y Sala-i-Martin (1992a; 1992b), se aplicó abundantemente al análisis de la convergencia regional en Estados Unidos, Canadá, Japón, Australia y países europeos (Alemania, Austria, Reino Unido, Francia, Italia y España), encontrando evidencia de convergencia absoluta y condicional (cercana al $2 \%$ en promedio) ${ }^{3}$. Un común denominador en estos resultados es la amplia dispersión en la velocidad de convergencia en cada país, lo que además indica cambios profundos en el patrón de convergencia a través del tiempo; la convergencia aparece fuerte al final de la década de 1970, se detiene durante la década de 1980, y luego se reactiva, pero a un ritmo lento. Las estimaciones son sensibles al conjunto de países, a la regionalización utilizada, y a las variables explicativas adicionales.

Las críticas a este análisis tradicional (AT) de corte transversal señalaron que estas ecuaciones resultaban compatibles con diferentes interpretaciones teóricas del proceso de crecimiento económico (crecimiento endógeno, difusión tecnológica, proximidad espacial y derramamientos, entre otros), por lo cual no constituían una verificación de la teoría neoclásica. También destacaban que la relación negativa entre la tasa de crecimiento y los valores iniciales del ingreso per cápita no necesariamente se presenta con una reducción en la varianza, con lo que es posible tener una distribución divergente en el corte transversal y un parámetro $\beta$ negativo. Estas posibilidades señalaban la necesidad de obtener más información sobre el comportamiento de toda la distribución del ingreso. De aquí que la convergencia beta se complemente con el cálculo de la convergencia sigma $(\sigma)$, que corresponde a una reducción en la evolución de la desviación estándar del corte transversal del logaritmo del ingreso per cápita 4 .

3 Este 2\% implica que a una economía le toma 35 años reducir, a la mitad, la brecha entre su producto per cápita inicial y su producto per cápita de largo plazo.

4 La convergencia sigma es una medida de la dispersión del ingreso con la cual se realiza un análisis exploratorio.
Una forma de tratar más adecuadamente la heterogeneidad no observada e incluir información dinámica es a través del uso de los métodos de datos de panel. En esta literatura, se aplican estimadores como el de la variable dummy de mínimos cuadrados (Hsiao, 1986), el estimador en dos etapas del método generalizado de los momentos (MGM) (Arellano, 1988; Arellano y Bond, 1991) y su estimador sistémico (Arellano y Bover, 1995; Blundell y Bond, 1998). Los resultados obtenidos por estos estudios son controversiales, debido a que la mayoría encuentra tasas de convergencia mucho más elevadas que las reportadas por las estimaciones de corte transversal (desde el 10\% hasta el 39\%). Además, al eliminar la heterogeneidad, estos cálculos siguen sin revelar cómo evoluciona la distribución de corte transversal de las economías.

Otro camino metodológico para verificar la convergencia es el de las series de tiempo. Uno de estos métodos, propuesto por Bernard y Durlauf (1995), modela la serie del producto de una economía como $\alpha(L) \mathrm{y}_{\mathrm{i}, \mathrm{t}}=\mu_{i}+\varepsilon_{i, t}$, en donde $\alpha(L)$ tiene raíz en el círculo unitario (estacionaria en primeras diferencias) y el término aleatorio se distribuye con media cero estacionariamente. Bajo este marco, es posible demostrar que la convergencia $\beta$ equivale a la convergencia estocástica del pronóstico de largo plazo de los productos de las economías, dado un conjunto de información $I_{t}$, la cual es igual a $k \rightarrow \infty$

$\lim _{k \rightarrow \infty} E\left(y_{i, t+k}-y_{p, t+k} \mid I_{t}\right)=0, \forall p \neq 1$.

Aunque esta noción de convergencia aparece más estricta que la utilizada en el análisis de corte transversal, otros autores han demostrado que la estacionariedad no es una condición necesaria para la convergencia, por lo cual este método fallaría en identificar si realmente esta última existe. Lo cierto es que ambos métodos, corte transversal y series de tiempo, arrojan resultados diferentes utilizando bases de datos similares.

\subsubsection{Interdependencia y efectos espaciales}

La interdependencia entre países y, más aún, entre regiones, que genera los flujos comerciales, además de los flujos de factores, plantea interrogantes sobre la validez del AT. A nivel teórico, el escenario neoclásico de Solow-Swan es ampliado a economías abiertas bajo los supuestos de tecnologías y preferencias similares y libre movilidad de factores; esta última en función de las 
tasas relativas de remuneración de los factores. Tales remuneraciones pueden diferir entre regiones o países y están regidas por mercados competitivos. Sin embargo, se asume que el mercado de capital no está integrado. En consecuencia, en este nuevo escenario se refuerza la convergencia neoclásica, arrojando velocidades mucho más altas que las de la economía cerrada.

En el ámbito de la convergencia regional, la velocidad también se incrementa, incluso si se restringe la movilidad de factores, pero se mantiene la libre movilidad de bienes. Mecanismos como la igualación de los precios de los bienes y la especialización productiva regional conducen a la igualación de los precios de los factores (Teorema de Samuelson), e incluso a la difusión tecnológica, aumentando la posibilidad de convergencia entre regiones pobres y ricas. Bajo estos supuestos, las economías regionales comparten el mismo estado estacionario, en donde el crecimiento económico es el resultado de dos fuerzas: a) el crecimiento internamente financiado a través del stock de capital por trabajador; b) la reducción progresiva de la asignación ineficiente de recursos regionales causada por la apertura comercial.

No obstante, las tasas de convergencia entre regiones reportadas en la literatura internacional, a partir de regresiones de corte transversal, son bastante bajas (cercanas al 2\%). Una respuesta para ajustar estos modelos ha sido incluir explícitamente el efecto de la tasa de migración, en tanto parte de los flujos interregionales. Algunos estudios para Estados Unidos, Japón y la Unión Europea reportan resultados mixtos. Según Magrini (2004), la literatura en convergencia regional no ha prestado mucha atención a la movilidad de los factores como un elemento importante para entender la dinámica del crecimiento regional.

Así mismo, la presencia de flujos interregionales introduce potenciales problemas en las especificaciones econométricas del AT: la dependencia espacial y la heterogeneidad espacial. La primera comprende dos tipos: puede ser sustantiva, es decir, causada por derramamientos tecnológicos o de movilidad de los factores; o distorsionante: debida a un error en la medición generada por el desajuste entre el patrón espacial analizado (ej. Integración de mercados) y la delimitación espacial observada (ej. Fronteras políticas). La heterogeneidad espacial se refiere a la inestabilidad general en el comportamiento a través de las unidades observadas.

Anselin (1988; 1995) y Anselin y Bera (1998), entre otros, han desarrollado un amplio conjunto de estimadores para detectar estos efectos espaciales. La extensión más sencilla de la estimación de corte transversal, para capturar la dependencia espacial sustantiva, incluye un rezago espacial de la variable dependiente. En este caso, la tasa de crecimiento del producto per cápita. La expresión por estimar es $(1 / t) \log [y(t) / y(0)]=c-\left(1-e^{-\beta t}\right) / t * \log y(0)+$ $\lambda_{1} W \log [y(t) / y(0)]+u(t)$,

donde $\lambda_{1}$ es un parámetro espacial autorregresivo y $W$ es la matriz de pesos espaciales estandarizada que describe la estructura e intensidad de los efectos espaciales. El término espacial en esta ecuación puede interpretarse como la autocorrelación en los ingresos iniciales, o la autocorrelación presente en el proceso de generación de datos, que surge cuando el crecimiento económico de una región se relaciona no solo con su propio nivel inicial, sino también con el crecimiento de otras regiones. Dada la presencia de dependencia espacial, el estimador de OLS es inconsistente, por lo que se utilizan los estimadores de máxima verosimilitud y de variables instrumentales ${ }^{5}$.

La dependencia espacial distorsionante, en un modelo de corte transversal, se incluye a través de un término de error no-esférico (el error no es constante ni invariante) de la forma: $\varepsilon(t)=\left(I-\lambda_{3} W\right)^{-1} u(t)$, donde $\varepsilon(t) \sim N\left(0, \sigma^{2}\right)$ y $\lambda_{3}$ son un coeficiente del error espacial. Para obtener estimaciones insesgadas de la varianza, se aplican estimadores de máxima verosimilitud o del método generalizado de los momen-

5 Modelo de rezago espacial (Spatial Autoregressive Model-SAR). En el cálculo de la convergencia $\beta$ condicional es posible incluir la dependencia espacial aportada por las variables de control a través del Spatial Durbin Model (SDM). Los coeficientes espaciales permiten estimar las externalidades espaciales globales. 
tos. En este caso, la externalidad se transmite a partir de shocks aleatorios que afectan a varias regiones a la vez ${ }^{6}$.

A comienzos de la década del 2000, los análisis de corte transversal con efectos espaciales eran relativamente pocos (Magrini, 2004; Rey y Janikas, 2005). Estos estudios sobre la convergencia regional en Estados Unidos y en regiones de Europa encuentran fuerte evidencia de efectos espaciales. Lo que destaca la incorrecta especificación del AT, debido a la omisión de variables espaciales. Además, encuentran que una vez la dependencia espacial es capturada, la velocidad de convergencia disminuye. Así, la presencia de dependencia espacial distorsionante requiere examinar con cuidado qué tipo de unidades espaciales son más apropiadas para el análisis empírico, ya que comúnmente se utilizan regiones definidas administrativamente que no siempre corresponden al patrón espacial del fenómeno económico. Es el caso de las regiones europeas denominadas NUTS (Nomenclature of Territorial Units for Statistics), definidas en diferentes niveles de agregación con una alta heterogeneidad en sus unidades territoriales. Una alternativa a estas fronteras regionales está en la clasificación FUR (Functional Urban Regions), cuyo concepto de base son las áreas estadísticas metropolitanas construidas inicialmente para los Estados Unidos.

La econometría espacial para paneles de datos es más complicada que aquella aplicada a datos de corte transversal y series de tiempo, ya que presenta dos principales dificultades de estimación, tanto en modelos de efectos fijos como aleatorios. Por un lado, la dependencia espacial puede darse entre observaciones en cada punto del tiempo. Por otro lado, se presenta heterogeneidad en los parámetros según la ubicación geográfica, lo cual contrasta con el supuesto de homogeneidad en parámetros según grupos, que usualmente se aplica en los

6 En el modelo de error espacial (Spatial Error Model-SEM), el coeficiente espacial se refiere a externalidades espaciales locales. Un modelo comprensivo de los anteriores es el Spatial Autoregressive Model with Autoregressive Disturbance (SARAR) (LeSage y Pace, 2009). modelos de panel (Elhorst, 2003). Incluso los parámetros pueden variar a través del tiempo, de lo cual se ocupan los modelos de paneles dinámicos (Badinger et al., 2004) ${ }^{7}$.

\subsubsection{Dinámica distributiva (DD)}

Debido a que el AT identifica la dinámica de la economía representativa y su transición a su propio estado estacionario, dejando de lado el comportamiento de toda la distribución de corte transversal del ingreso, el enfoque distributivo de la convergencia surge como un método alternativo.

Quah (1993; 1996a; 1996b; 1997) explicó que la convergencia $\beta$ era necesaria, pero no suficiente, para lograr la convergencia $\sigma$, por lo que una desviación estándar constante podría ser compatible con dinámicas muy diferentes. La dinámica distributiva (DD) expande la información de la convergencia $\sigma$ con el uso de Kernels estocásticos para capturar la evolución en el tiempo de la distribución del ingreso. La expresión más sencilla para modelar esta dinámica es una especificación de dependencia de primer orden, en donde $\phi_{t}$ es una probabilidad asociada a $F_{t}$, que denota la distribución de corte transversal en el tiempo t, así: $\phi_{t}=T^{*}\left(\phi_{t-1}, u_{t}\right)=T_{u_{t}}^{*}\left(\phi_{t-1}\right)$, donde $u_{t}$ es una secuencia de errores, $T^{*}$ es un operador que mapea el producto cartesiano de probabilidades en $t-1$ y errores en $t$, mientras que $T_{u_{t}}^{*}$ es un operador que ahora incorpora el error $y$ codifica información de la dinámica al interior de la distribución.

En un contexto discreto, y asumiendo un mecanismo de transición invariante en el tiempo (cadena de Markov homogénea y finita), la iteración de esta probabilidad produce un predictor de las futuras distribuciones de corte transversal: $\phi_{t+s}=M^{\prime}$ 's $\phi_{t}$, en donde $M^{\text {'s }}$ es una matriz de probabilidades de transición que, aplicada al análisis de la convergencia, indica la probabilidad de moverse entre dos clases de ingreso en $s$ periodos de tiempo (enfoque paramétrico). Solo en el caso en que $\phi_{t+s}$ exhiba una tendencia a concentrarse en un punto de

7 Ver desarrollos recientes en econometría espacial en Fischer y Getis (2010). 
masa, existe convergencia; si por el contrario la tendencia se concentra en dos puntos, el fenómeno es de polarización.

Las críticas a este método indicaban la pérdida de información por la discretización del ingreso, y también por los supuestos al proceso generador de datos impuesto por la cadena de Markov. En respuesta, Quah (1996a; 1996b) encontró que una alternativa es el enfoque no-paramétrico, en donde se analiza directamente la forma del gráfico tridimensional del kernel estocástico $\left(\phi_{t}\right)$.

Los resultados de este método son mixtos en el caso de Estados Unidos, ya que la evidencia de convergencia con kernel estocásticos paramétricos es contradictoria. Cálculos para la Unión Europea, que utilizan las regiones NUTS, arrojan persistencia en los rangos de bajos ingresos, mientras que, si se utilizan las regiones FUR, la evidencia es de polarización. La aplicación de kernel estocásticos no-paramétricos ofrece resultados de convergencia en los Estados Unidos y de persistencia en la Unión Europea (Magrini, 2004).

Una vez se identifica la forma y la DD de la distribución de corte transversal del ingreso, queda el interrogante sobre sus factores explicativos. En esta dirección, Quah (1997) propone la noción de clubes de convergencia, a través de la introducción de un esquema condicionante en la distribución del ingreso, en donde se asocia el comportamiento de una región con el de sus vecinos circundantes.

Al igual que en el AT, los factores espaciales también aparecen para cuestionar los supuestos de independencia y homogeneidad entre unidades observadas. La matriz espacial de Markov es la versión modificada de la matriz tradicional en la que se condiciona la probabilidad de transición de una región al nivel de ingreso de sus vecinos. Otro desarrollo está en la estimación de kernel estocásticos y la aplicación del esquema condicionante del ingreso de Quah (1996b), para evaluar el efecto de la interacción espacial entre regiones contiguas. Aplicaciones de este método indican que la interacción espacial local es un factor fundamen- tal para explicar la desigualdad en ingresos en las regiones europeas (Fischer y Stumpner, 2010).

\subsection{Extensiones del análisis neoclásico}

La teoría del crecimiento endógeno (Aghion y Howitt, 1998; Lucas, 1988; Romer, 1986) y la Nueva Geografía Económica (NGE) (Fujita et al., 1999) proporcionan bases conceptuales más amplias, que las del modelo de crecimiento neoclásico tradicional, para comprender las dinámicas diferenciadas del crecimiento económico entre países y entre regiones. En el marco del crecimiento endógeno, la tecnología es una variable explicativa, usualmente medida a través del capital humano, que experimenta procesos de trasferencia y de derramamiento espacial. A su vez, la NGE explica los procesos de aglomeración a partir de rendimientos a escala crecientes y de fenómenos de competencia imperfecta, los cuales determinan una distribución espacial de la actividad económica heterogénea y con fijaciones locacionales.

Existen diversos modelos dentro de la teoría del crecimiento endógeno que explican los mecanismos de la creación y acumulación de conocimiento. Por ejemplo, en Lucas (1988) se plantea que los derramamientos de conocimiento (knowledge spillovers) están geográficamente circunscritos debido a que ellos están incorporados en el capital humano (personas), siendo su proximidad e intercambio directo determinantes. En consecuencia, la dinámica de acumulación del capital humano es esencialmente regional. Otro elemento que modifica el análisis neoclásico está en los rendimientos crecientes a escala, provenientes de la endogeneización de la creación y acumulación de conocimiento, los cuales afectan la tasa de crecimiento de largo plazo.

En Estados Unidos, una parte importante de las aplicaciones de las teorías del crecimiento endógeno se concentró en la economía urbana, utilizando como unidad de medida áreas metropolitanas (Standard Metropolitan Statistical Areas -SMSA-) y como variable dependiente el crecimiento en el empleo. Es así como Roberts 
y Setterfield (2010) destacan los estudios de E. Glaeser, en los cuales se verifica la importancia del capital humano especializado para el crecimiento regional, así como los potenciales efectos de la especialización o la diversificación productiva y la competencia de mercado.

En Europa, los estudios se han orientado hacia el crecimiento regional con base en las regiones NUTS, que son unidades menos desagregadas y contienen un mayor error de medición. Estos encuentran la no convergencia entre regiones desde mediados de la década de 1970 y destacan la importancia del capital humano y su acumulación, indicando que, ante derramamientos de conocimiento muy fuertes, la divergencia es inevitable (Roberts y Setterfield, 2010).

No obstante, el vínculo entre esta teoría y la modelación empírica del crecimiento regional ha sido débil, ya que existen problemas de identificación y la literatura sobre convergencia regional ha sido lenta para desarrollar modelos de cambio y difusión tecnológica con un componente espacial explícito, utilizando apenas una pequeña fracción de todos los modelos econométricos disponibles (Rey y Janikas, 2005; Roberts y Setterfield, 2010). Por ejemplo, Fingleton y López-Bazo (2006) observan que una parte importante de los estudios que estiman modelos empíricos del crecimiento económico con efectos espaciales no aplican los modelos de rezago espacial, que capturan la principal fuente de la dependencia espacial (sustantiva), y representan las externalidades analizadas por la NGE y la teoría del crecimiento endógeno. En su lugar, la tendencia dominante en este grupo de estudios está en el uso de los modelos de error espacial que suponen una dependencia espacial distorsionante.

\subsection{Convergencia social}

Ante la controversia en los resultados empíricos alrededor de la hipótesis de convergencia en ingresos, algunos estudios proponen la inclusión de indicadores de calidad de vida como una medida no-monetaria y complementaria del ingreso. Becker et al. (2005) calculan el valor monetario del incremento en longevidad y lo adicionan al incremento en PIB per cápita para obtener una medida del ingreso total. Usando una serie de corte transversal con 96 países para los años 1960, 1990 y 2000, estos autores encuentran un resultado contrario al diagnóstico de incremento en la inequidad entre países: los ingresos totales per cápita convergen entre 1960 y 2000 . Otra evidencia de convergencia entre bloques de países a nivel mundial se encuentra utilizando el Índice de Desarrollo Humano (IDH), el cual pondera el ingreso per cápita con variables como la expectativa de vida al nacer y el alfabetismo. El IDH se utiliza para medir el crecimiento de largo plazo de la calidad de vida (living standards), revelando un crecimiento notablemente positivo para los países menos desarrollados a partir de 1950 (Crafts, 2000). En este contexto, el ingreso monetario es una medida inadecuada e incompleta del bienestar material y en general del desarrollo, el cual debe ser multidimensional e incluir aspectos como salud, actividades por fuera del mercado, ocio y desigualdad.

Una parte de estos estudios dejan de construir medidas ponderadas del ingreso total para calcular la convergencia directamente sobre diversos indicadores en salud (expectativa de vida, mortalidad infantil, calorías consumidas per cápita), educación (alfabetismo, cobertura en educación primaria, años de educación), y contexto político (derechos civiles, libertades políticas, muertos en guerra). La convergencia $\sigma$, la DD y la convergencia $\beta$ son los métodos aplicados para efectos exploratorios y confirmatorios de la hipótesis de convergencia. Con esta base, Kenny (2005) revindica los logros de la política multilateral de desarrollo, al observar que los esfuerzos de los países en desarrollo han sido malogrados si se mira solamente el desempeño del ingreso monetario. De aquí se desprende que, si el ingreso no es tan importante en el avance de la calidad de vida, tampoco lo son las transferencias, luego el enfoque de la política tendría que centrarse en mejorar variables vitales como la salud y la educación. En otras palabras, en garantizar el acceso (en especie) a los servicios básicos.

Aunque la evidencia encontrada en diversos países a través de ejercicios de corte trans- 
versal y panel de datos es confirmatoria, el sustento teórico que explicaría el mecanismo por el cual la expectativa de vida o el acceso al agua convergen no es explícito. Este sustento tendría que mostrar cómo aumentos muy pequeños en el ingreso monetario se acompañan de ganancias sustanciales en indicadores sociales, y cómo se intervinculan diversas variables sociales cuyo efecto no es fácilmente medible (ej. libertad económica, concentración del poder político) ${ }^{8}$.

\section{Estudios sobre la}

\section{convergencia regional en Colombia}

La convergencia regional es el concepto a través del cual los economistas colombianos se vincularon al estudio de las disparidades económicas regionales, en especial desde mediados de la década de 1990. Dos razones motivan la investigación: por un lado, los trabajos de Barro (1989) y Barro y Sala-i-Martin (1991; 1992a; 1992b) y, por el otro, la descentralización, política, administrativa y fiscal que inicia a mediados de 1980, afianzada a partir de la Constitución política de 1991 y orientada a reducir las brechas regionales.

A continuación, se identifican los estudios más relevantes publicados entre 1993 y 2018 , presentados de acuerdo con los grupos de metodologías definidos en la sección anterior. Debido a que algunos estudios combinan métodos, este agrupamiento no es tan estricto; los artículos que combinan dos metodologías se clasifican según aquella de más reciente desarrollo, y aquellos con más de dos métodos se presentan en una sección aparte. Además del método aplicado, la revisión privilegia las

8 Mientras la hipótesis de convergencia en ingresos se apoya en el modelo de crecimiento neoclásico, la hipótesis de convergencia social carece de un sustento similar. La base teórica para conectar el proceso de crecimiento económico con un proceso de cierre de brechas sociales es más incierta, siendo la curva de Kuznets el referente más destacado y al mismo tiempo el más cuestionado. Ver discusión en Meisel y Galvis (2009). mediciones de la variable dependiente, el período de estudio y sus principales resultados. Solo en algunas ocasiones se mencionan otros detalles adicionales como las variables de control.

\subsection{Análisis tradicional y análisis espacial}

Cárdenas et al. (1993) y Cárdenas y Pontón (1995) fueron los pioneros en el AT. La estimación para su periodo de análisis (1950-1989) los lleva a concluir que Colombia es un caso exitoso de convergencia en PIB per cápita departamental, al hallar convergencia $\beta$ absoluta $y$ significativa de 4,2\%. Este resultado desató una discusión en los estudios posteriores.

El primer estudio que bajo la misma metodología lo confronta es el de Bonet y Meisel (2001), usando un periodo más largo de análisis (19261995), dividido en dos subperiodos para analizar la convergencia $\beta$ y $\sigma:$ 1926-1960 y 1961 1995. Los datos corresponden a los depósitos bancarios per cápita como proxy del nivel de ingreso departamental en el primer periodo y al PIB per cápita real departamental para el segundo. Para el primer período, los autores encuentran la existencia de convergencia $\beta$ absoluta y significativa de $2,5 \%$ y una tipo $\sigma$, que se reduce de $1,25 \%$ en 1926 a $0,66 \%$ en 1960 . Esta convergencia se explica porque el país recibe por primera vez considerables flujos de ingresos por la indemnización de Panamá en 1920, que se invierten en infraestructura ferroviaria y malla vial, permitiendo una mejor integración entre regiones. En el segundo período los resultados son distintos, ya que los autores encuentran evidencia débil de convergencia $\beta$ absoluta de $1,3 \%$ no significativa. En cuanto a la convergencia $\sigma$, se concluye que esta se mantuvo relativamente estable entre 1960 y 1975; sin embargo, a partir de 1980, hay un aumento de la dispersión y un proceso de polarización en el PIB per cápita departamental. La explicación radica en los efectos de las políticas de industrialización por sustitución de importaciones (ISI), la consolidación de Bogotá como la gran metrópoli colombiana y el declive económico de los departamentos caribeños. Meisel (1993) ofrece una discusión al respecto. 
Un trabajo único por su metodología para el momento en que se encontraba la literatura en el país es presentado por Rocha y Vivas (1998). Esta investigación estudia los determinantes y patrones de crecimiento regional en Colombia entre 1980 y 1994. Su aporte metodológico consiste en aplicar una predeterminación bayesiana. Modificando la ecuación del AT, los autores hacen endógena la política fiscal, la inestabilidad sociopolítica, la fertilidad y el funcionamiento de los mercados; además, trabajan con heterogeneidad en las preferencias y la tecnología. El resultado es la verificación de la hipótesis de persistencia de la desigualdad en el periodo analizado, al estimarse la distribución de las trayectorias de largo plazo en función de la educación, las transferencias municipales y el acceso al crédito. Con relación a las transferencias, la estimación muestra que están relacionadas negativamente con el crecimiento del PIB departamental, lo que significa que ese gasto social no ha contribuido a una mejor equidad en el PIB. Cabe señalar que la velocidad de convergencia hallada con esta metodología es del 39\%, muy superior al 2 - 4\% del AT. Aun así, el patrón sigue siendo de persistencia en la desigualdad del PIB per cápita departamental.

Otro estudio que, para el momento, representó la primera aplicación del AT con datos de panel es presentado por Soto (1998). El autor encuentra la ausencia de convergencia $\beta$ absoluta entre 1960 y 1995 . No obstante, halla convergencia condicional de acuerdo con las variables de PIB per cápita inicial, educación secundaria, variable sectorial y crecimiento poblacional. En cuanto a la convergencia $\sigma$, no hay evidencia que muestre una disminución progresiva en la dispersión de los ingresos departamentales. Ese mismo resultado, utilizando series de tiempo, es confirmado por Rhenals et al. (1998), quienes encuentran ausencia de convergencia $\beta$ absoluta para todo el periodo 1960-1995. No obstante, al separar por subperiodos (1960-1975 y 1980-1995) y agrupar por departamentos, en el primer subperiodo encuentran una velocidad de convergencia condicional muy lenta $(0,3 \%) \mathrm{y}$, en el segundo, un claro proceso de divergencia.
Galvis y Meisel (2001) analizan la convergencia para las 20 principales ciudades del país y hacen uso de los depósitos bancarios per cápita reales como proxy del PIB per cápita. No encuentran un proceso de reducción de disparidades entre regiones entre 1973 y 1998. Además, encuentran una velocidad de convergencia $\beta$ absoluta de $-1,8 \%$ significativa, que sugiere un leve proceso de divergencia. También condicionan el modelo de regresión con capital humano, dotación de infraestructura y calidad de las instituciones, y encuentran una muy baja convergencia $\beta$ condicionada entre $0,03 \%$ y $0,7 \%$.

Barón y Meisel (2003) enfatizan en el rol de la Constitución de 1991, ya que su estudio solo aborda la década de 1990 y describe el proceso de descentralización fiscal que ocurrió con la nueva carta constitucional. Los autores concluyen que no existió convergencia $\beta$ en el ingreso per cápita de los departamentos, y que tampoco se puede hablar de convergencia $\sigma$ en ese periodo, reafirmando que las disparidades regionales tendieron a aumentar. Ante este panorama, los autores proponen un mecanismo que denominan de reducción de la inequidad fiscal interterritorial, mediante un fondo de compensación progresivo que transfiriera recursos de las regiones más ricas hacia las más pobres. Por su parte, Barón (2004) reafirma el mismo resultado obtenido en el estudio anterior para la década de 1990. La diferencia es que esta vez no menciona el papel de la nueva constitución en ese proceso divergente y, además, incluye la década de 1980 dentro de su período de análisis, en donde sí encuentra evidencia de convergencia $\beta$ absoluta de $4,1 \%$ significativo; esto resulta paradójico si se tienen en cuenta los trabajos discutidos hasta el momento. Al tomar todo el periodo, 1980-2000, la evidencia señala ausencia de convergencia $\beta$ absoluta. Para convergencia $\sigma$ se encuentra el mismo patrón de resultados: existió en la década de 1980, no la hubo en la década de 1990 y está ausente en todo el periodo.

Acevedo (2003) estudia este proceso entre 1980 y 2000 y concluye que, para las dos décadas en conjunto y los años 80 , hubo convergencia $\beta$ condicionada en grupos de departamentos con 
niveles similares de ingresos; en contraste, para la década del 90 no existe evidencia a favor de la convergencia condicional. Por su parte, Franco y Raymond (2009) usan un panel de datos con efectos fijos para definir grupos o clubes regionales de convergencia. Sus resultados sugieren que Colombia ha venido experimentando un proceso de polarización en el PIB per cápita departamental, es decir, la ausencia de convergencia $\beta$ no condicional y convergencia $\sigma$. Por el contrario, se observa la presencia de convergencia condicionada en clubes departamentales.

Bonet y Meisel (2007) usan datos del Sistema Simplificado de Cuentas Departamentales (SSCD) del CEGA (Centro de Estudios Ganaderos y Agrícolas) para medir la convergencia $\sigma$, utilizando el Ingreso Departamental Bruto per cápita (IDBpc), entre 1975 y 2000. Los resultados no son concluyentes: por un lado, la dispersión se reduce levemente con la desviación estándar, pero es estable con el índice de Theil. Al ponderar demográficamente la desviación estándar, la dispersión aumenta, con lo cual los autores afirman que la única forma para que la tendencia sea convergente es excluir del cálculo a Bogotá. Este análisis es más de tipo exploratorio que confirmatorio.

Branisa y Cardozo (2009b) trabajan con dos mediciones del CEGA en el periodo 1975-2000: el Producto Per Cápita Departamental Bruto (PDB) y el Ingreso Per Cápita Bruto Disponible de los Hogares (IDBH). Los resultados indican que para las dos mediciones no hay evidencia de convergencia $\beta$ condicionada ni $\sigma$. Para el PDB encontraron una velocidad de convergencia $\beta$ absoluta de $0,7 \%$ no significativo y, para este mismo indicador, el IDBH fue de 1,2\% significativo al $5 \%$, es decir, existe evidencia a favor de la convergencia en el ingreso disponible.

Galvis et al. (2017) ofrecen una revisión de los principales estudios sobre la convergencia regional en Colombia en términos de PIB per cápita, identificando las metodologías utilizadas (AT y su extensión en series de tiempo, la DD y la inclusión de efectos espaciales). Los autores realizan varios cálculos de la convergencia en ingresos durante el período 1995-2015, para finalmente concluir que la distribución del ingreso regional ha sido divergente y persistente. En su análisis, se destaca la construcción de diferenciales de ingreso por regiones, y el cálculo de las tasas de crecimiento requeridas para que las regiones con menores niveles de PIB per cápita cierren la brecha en ingreso respecto de las más ricas.

La investigación más reciente sobre convergencia en Colombia es presentada por HahnDe-Castro y Meisel (2018). Los autores construyen una serie de tiempo a través de tasas de crecimiento del PIB per cápita para seis regiones colombianas, en un periodo de noventa años (1926-2016). En este aspecto este trabajo sobresale, ya que considera a la unidad territorial como un agregado de departamentos o conjunto de regiones. De esta manera, aplican el AT en su extensión en series de tiempo. Respecto a la convergencia $\beta$ absoluta dan cuenta de un proceso convergente, por medio de la relación entre el PIB per cápita inicial de cada región y su tasa de crecimiento observada durante el periodo de estudio. Esta relación es negativa. Sin embargo, presenta un bajo grado de significancia estadística que se pierde cuando se excluyen los antiguos territorios nacionales. En cuanto a convergencia $\sigma$ se concluye que, hasta 1960, no existe un patrón claro de aumento o disminución de la dispersión de la riqueza; no obstante, en los siguientes 50 años, este indicador ha aumentado de 0,19 en 1960 a 0,82 en 2016. Finalmente, en un marco de series de tiempo, donde el análisis se lleva a cabo únicamente por pares de regiones para detectar la presencia de raíces unitarias, se concluye que hay poca evidencia a favor de la hipótesis de convergencia regional.

Por su parte, Galvis y Meisel (2013) estiman un $\beta$ absoluto de $2,1 \%$ entre 1990 y 2010 que, si bien indica un leve proceso de convergencia, los autores cuestionan, ya que el proceso se ha dado solamente entre regiones iguales, siendo la dispersión global más alta a través del tiempo. Una de las novedades en este estudio es la introducción del análisis espacial, a través de un enfoque exploratorio aplicado a la pobreza y medido con el Índice de Moran. Con base en estos resultados, los autores sostienen que es erróneo comparar regiones con altos niveles de pobreza respecto a las de baja 
pobreza, indicando además que las regiones rezagadas no son las que reciben las mayores transferencias del gobierno central.

Una investigación que aplica el AT extendido teóricamente e incluye los efectos espaciales, ahora en un análisis confirmatorio, es presentada por Galvis y Hahn (2016). Los autores evalúan la hipótesis de convergencia $\beta$ absoluta y condicional, empleando los ingresos tributarios y depósitos bancarios municipales como proxy del PIB per cápita para el período 1993-2012. En el marco de un modelo de crecimiento endógeno en el cual se incluye el capital físico y humano, se incorporan sus externalidades espaciales por medio del modelo espacial de Durbin (SDM) y el modelo de error espacial (SEM). La estimación del AT da como resultado un $\beta$ del $2 \%$, y cuando se incluye el rezago espacial, el $\beta$ arroja un valor del 3,6\%. Sin embargo, para interpretar el efecto espacial sobre la velocidad de convergencia es necesario hallar el efecto directo y el efecto indirecto. El primero indica que las municipalidades más pobres crecen más rápido que las más ricas; mientras que el segundo evidencia que el vecindario de estos municipios pobres estanca su crecimiento, al contrario de lo que sucede con los más prósperos. Debido a que el efecto indirecto prevalece sobre el directo, se concluye que en Colombia no ha existido un proceso de convergencia condicional y se refuerza la evidencia sobre clubes de convergencia. Los autores destacan el papel de la inversión en capital físico y humano, y sus derramamientos espaciales a través de procesos de difusión tecnológica, como factores determinantes en el crecimiento municipal.

\subsection{Dinámica distributiva}

Birchenall y Murcia (1997) son pioneros en la aplicación de la DD con Kernel estocástico, el cual permite representar de forma dinámica la evolución en el tiempo de una variable y sus movimientos internos (persistencia, movilidad, convergencia o divergencia). Los autores encuentran que, entre 1960 y 1994 , hubo una gran persistencia en la distribución de los ingresos entre los departamentos, ya que su posición del año inicial respecto al año final de análisis se sostiene. Cabe resaltar que este trabajo se enfocó, en su momento, en presentar una alternativa a la medición tradicional de la convergencia.

Ardila (2004), usando datos del CEGA para PIB per cápita departamental, analiza un periodo de diez años entre 1985 y 1996, caracterizado por una alta persistencia en la distribución del ingreso entre departamentos. Esto se explica a través de la evolución del gasto público, especialmente la inversión, la cual ha evitado la polarización entre los departamentos, impidiendo que regiones con alto PIB per cápita como Bogotá se alejen aún más del resto. Esto implica que se ha afectado la posición relativa de algunos departamentos debido a la inversión pública, sin embargo, la dinámica de la distribución del ingreso no ha variado. Ardila (2004) señala que es necesario evaluar si al menos ese incremento en el gasto público, y especialmente en la inversión, ha tenido un efecto más significativo sobre la distribución regional del ingreso que el señalado en su trabajo.

Como se mencionó, el trabajo de Bonet y Meisel (2007) calcula la convergencia $\sigma$. Sin embargo, este cálculo se complementa estimando la función de densidad de Kernel para los años $1975,1980,1990$ y 2000 con base en datos del CECA del IDBpc. Los autores observan una polarización en el IDBpc con dos polos que ampliaron sus diferencias en el tiempo. Bogotá se ubica en el extremo superior, alejándose cada vez más de la media nacional, y el resto de departamentos converge a la media nacional. Finalmente, la dinámica es de persistencia en la distribución durante todo el período 1975-2000. En la misma línea, están Branisa y Cardozo (2009b), quienes complementan el AT con el método de la DD. Con este último, los resultados indican que para el PDB el patrón es de persistencia, mientras que para el IDBH es de decrecimiento en su dispersión durante el período 1975-2000.

\subsection{Convergencia en indicadores sociales}

Uno de los retos que han tenido los investigadores, a la hora de estudiar la convergencia a un nivel más desagregado, es la disponibilidad 
de cifras sobre PIB municipal. De aquí el giro hacia el uso de indicadores sociales y otras variables proxy del ingreso que reflejarían la mayor producción de datos desagregada en el país, incentivando este tipo de estudios y la aplicación de técnicas cuantitativas sofisticadas. Con este nuevo paso, la hipótesis de convergencia en ingresos se aplicaría a la convergencia en indicadores sociales, planteando que aquellas regiones con niveles sociales más desfavorables mejorarían más rápido su calidad de vida respecto a aquellas con mejor situación social.

Dentro de los artículos pioneros están Aguirre (2005), Martínez (2006) y Branisa y Cardozo (2009a), los cuales toman indicadores en salud y educación del Departamento Administrativo Nacional de Estadística (DANE), medidos entre 1973 y 2005 . Aplicando los métodos del AT y DD, los dos primeros trabajos no hallan evidencia de convergencia para el indicador de educación (tasa de analfabetismo); por otro lado, verifican una tendencia a converger de la tasa de mortalidad infantil. Aguirre (2005) también encuentra convergencia en la esperanza de vida al nacer. Al contrario de estos resultados, Branisa y Cardozo (2009a) hallan convergencia en los indicadores de educación, aunque no en los indicadores de salud. La diferencia radica en la eliminación de valores extremos en el análisis desarrollado por estos últimos. Además, en Branisa y Cardozo (2009a), todas las variables se expresan como una proporción del valor nacional y utilizan tasas de alfabetización, mientras que Aguirre (2005) usa tasas de analfabetismo. Martínez (2006) profundiza en la DD y deduce que la cobertura en educación básica y la tasa de mortalidad infantil tienen una distribución que ha disminuido su dispersión; caso contrario sucede con el PIB per cápita.

\subsection{Estudios multi-método}

Dentro de los estudios que combinan tanto el AT y la DD con efectos espaciales, y analizan la convergencia en términos de ingresos e indicadores sociales, están Loaiza y Moncada (2013) y Royuela y García (2015). Los primeros dos autores utilizan la herramienta del análisis exploratorio de datos espaciales (AEDE) para analizar la distribución espacial de la pobreza medida por el índice de necesidades básicas insatisfechas (NBI) de los censos de 1993 y 2005. De igual forma aplican el AT para identificar la convergencia $\beta$ absoluta y luego incluir explícitamente la dependencia espacial, para lo cual aplican modelos de error espacial y de rezago espacial, así como su combinación. Para esto utilizan los ingresos tributarios per cápita a nivel municipal de las cuentas fiscales del Departamento Nacional de Planeación (DNP) como proxy del PIB municipal entre 1985 y 2010.

Loaiza y Moncada (2013) encuentran que las municipalidades ubicadas al interior del país tienen una correspondencia alta y negativa entre el nivel de actividad económica y de pobreza. Esto se explica por la cercanía a las principales áreas de influencia de Bogotá y Medellín; situación contraria se experimenta en zonas de la costa caribe y pacífica. Esto ha permitido un proceso de contagio y difusión en las municipalidades cercanas a vecindarios de baja pobreza, que ha reducido brechas sociales en el Ilamado "Triángulo de Oro" (Bogotá, Medellín y Cali), no obstante, para el resto de las municipalidades periféricas no se puede afirmar lo mismo. Igualmente, los autores identifican al modelo de error espacial como el de mejor desempeño con el que calculan una convergencia no condicional en ingresos tributarios per cápita del $3,3 \%$. Esta velocidad es superior a la convergencia estimada con un AT sin efectos espaciales.

Royuela y García (2015) ofrecen un análisis diverso a partir de datos departamentales para el período 1975-2005, con los cuales investigan la convergencia $\beta$ absoluta y $\sigma$ en ingresos con base en el PIB per cápita y el ingreso disponible del hogar (IDBpc), y la convergencia social, utilizando indicadores de desarrollo humano tales como la tasa de alfabetismo, la expectativa de vida al nacer, la tasa de supervivencia al nacer, y la tasa de homicidios. Los métodos aplicados son el AT en corte transversal y en panel de datos, sin y con efectos espaciales (modelo espacial de error y el modelo de rezago espacial), así como la DD. Los autores combinan el análisis exploratorio, basado en el cálculo de la autocorrelación espacial de 
las series a través del estadístico I de Moran, el coeficiente de variación para la convergencia $\sigma$, y las estimaciones del kernel, con el análisis confirmatorio, basado en las estimaciones de los modelos econométricos.

Royuela y García (2015) encuentran un proceso divergente en PIB per cápita, aunque convergente en ingreso disponible de los hogares. Diferencia que explican a partir del efecto en producción de los booms mineros sobre algunos departamentos. En cuanto a los indicadores sociales, se evidencia convergencia en la tasa de alfabetismo, la esperanza de vida al nacer y la tasa de homicidios; los resultados son ambiguos respecto a la tasa de supervivencia al nacer, explicado en parte por los valores extremos del Chocó. Precisamente la heterogeneidad en el comportamiento de ciertos departamentos sirve a los autores para explicar el débil vínculo entre los efectos espaciales y las dinámicas de convergencia, y para reconocer la necesidad de hallar evidencia más robusta.

\section{Discusión}

A través de la revisión de la literatura internacional, se evidencia que al análisis de convergencia regional se aplican las mismas metodologías de AT y DD utilizadas en el examen de la convergencia económica entre países. Si bien la noción de convergencia se afina al pasar de la versión absoluta a la condicional, y los avances en el refinamiento del método y la medición contribuyen a aproximar mejor la magnitud y establecer las dinámicas de la convergencia regional, solamente con la inclusión de los efectos de interdependencia y los efectos espaciales es posible construir una conceptualización más apropiada de las realidades de las regiones subnacionales.

Los cálculos de las velocidades de convergencia regional en diversos países industrializados también muestran que estos son sensibles al método y a las mediciones. No obstante, varios estudios coinciden en que a partir de mediados de la década de 1970 estas velocidades disminuyen, resultado que algunos autores atribuyen al postfordismo o a shocks exógenos en la economía global (Martin y Sunley,
1998). A partir de este cambio de tendencia, la existencia de clubes de convergencia recibe más respaldo empírico.

A partir de las teorías del crecimiento endógeno y la NGE, esta literatura renueva el debate sobre los determinantes y mecanismos de la convergencia económica al incorporar bases teóricas más amplias para dar cuenta de los procesos de generación y difusión tecnológica, así como de aglomeración económica y externalidades. Sin embargo, los estudios empíricos que aplican econometría espacial estándar han dominado, mientras que el desarrollo de modelos de crecimiento endógeno con efectos espaciales ha sido lento. Autores como Roberts y Setterfield (2010) y Martin y Sunley (1998) indican que, para avanzar en la investigación sobre disparidades en el crecimiento regional, en un marco de crecimiento endógeno, es necesario incorporar el comportamiento de la demanda, y no solamente el de la oferta, para comprender fenómenos como los retornos crecientes localizados o la naturaleza de los derramamientos de conocimiento.

Así mismo, se debe reconocer explícitamente el carácter abierto de las economías, en este caso las regionales, las cuales conforman un sistema interrelacionado de vínculos positivos y negativos entre sí. De importancia fundamental también es reconocer la diversidad en el espacio y el tiempo de los patrones y mecanismos de crecimiento regional, indicados por ejemplo en los estudios sobre derramamientos al interior y a través de las industrias. Bajo esta luz, no existiría un único mecanismo de crecimiento endógeno que explicara todas las diferencias espacio-temporales en tasas de crecimiento, sino que más bien habría varios mecanismos operando cuyo efecto neto dependería del contexto (Roberts y Setterfield, 2010).

Mientras el cálculo de la convergencia social ha sido una alternativa para contrarrestar el pesimismo generado por la no convergencia en ingresos a nivel mundial y subnacional, es un hecho que la población más pobre del mundo se ha beneficiado de los avances en medicina y salubridad, aumentando su ex- 
pectativa de vida. Estos resultados pertenecen al análisis exploratorio y no confirmatorio que, como se anotó, carecen de fundamento teórico y reclaman una mayor conexión con las contribuciones de la literatura sobre inequidad, desarrollo y crecimiento económico.

La literatura aplicada para Colombia indica, en términos metodológicos, que el AT en corte transversal ha sido el método predominante en sus dos versiones de convergencia absoluta y condicional. El análisis de DD influenció a menos autores, y el análisis de efectos espaciales apenas ha recibido el debido tratamiento en unos pocos estudios. Las dificultades planteadas por la disponibilidad de datos, en especial la ausencia de series de tiempo congruentes y desagregadas sobre PIB y/o ingresos per cápita, están dentro de los factores que explican este lento avance en los métodos. De la misma forma, se encuentra una lenta entrada a nivel teórico de los modelos del crecimiento endógeno y la NGE.

Un balance sobre los resultados en convergencia en ingresos $\beta$ absoluta y condicional es el siguiente: Colombia fue un éxito de convergencia $\beta$ absoluta hasta 1970 y a partir de la década de 1990 presenta divergencia. En cuanto a la convergencia $\beta$ condicional, es posible observar diversidad en las variables de control según los distintos autores, lo que implica que las velocidades de convergencia cambien. En términos generales, las velocidades de convergencia $\beta$ absoluta oscilan entre el $2 \%$ y el $4 \%$ y, cuando se condicionan, entre el $1 \%$ y el $3 \%$. Un punto en común en los estudios está en demostrar que no todas las regiones parten del mismo punto inicial de crecimiento. En cuanto a los patrones de convergencia $\sigma$, se tiene que disminuyó la dispersión en el periodo 1926-1960, aumentó en el periodo 1990-1998, se estancó en la primera parte de la década del 2000 y aumentó a partir del 2005. En contraste, no existe consenso frente al patrón de la década de 1970 y 1980. Además, con la inclusión de la DD en el análisis, algunos

9 Royuela y García (2015) encuentran que la dispersión en el ingreso disponible de los hogares es baja y estable entre 1975-1985, pero aumenta a partir de mediados de la década de 1980 implicando divergencia. trabajos indican patrones de agrupamiento y polarización, evidenciando la existencia de clubes de convergencia. Sobre el componente espacial, no existe consenso sobre su efecto en el crecimiento regional colombiano ya que mientras para unos autores este es negativo, para otros es positivo (aunque débil).

Respecto a la convergencia social, los autores colombianos siguieron la tendencia internacional al analizar indicadores sociales a través del AT y la DD, incluyendo los efectos espaciales. Los estudios publicados, a partir del 2010, identifican espacialmente los clusters de pobreza y coinciden en un resultado paradójico: cierre de brechas sociales, pero permanencia o aumento de la brecha de PIB per cápita. A pesar del optimismo o mayor énfasis que la convergencia social puede suscitar, y de la alternativa que representa ante la ausencia de datos monetarios más desagregados o robustos, es importante ser conscientes del limbo teórico de este análisis.

Ante la débil convergencia regional y la polarización en las regiones colombianas, presentes al menos desde la década de 1990, ¿cuáles han sido las recomendaciones de política pública? Un punto focal es el activismo en la política de desarrollo regional para el cierre de brechas, y así una renuncia a la expectativa de igualación automática de ingresos en el largo plazo del mecanismo neoclásico. Este Ilamado a la intervención estatal tiene lugar a través de mayor inversión pública en infraestructura y educación, y de una asignación más efectiva del gasto descentralizado, cuyo efecto nivelador ha sido cuestionable, en especial en relación con las transferencias (Branisa y Cardozo, 2009b; Galvis y Meisel, 2013; Rocha y Vivas, 1998). Es así como los estudios realizados por investigadores del Banco de la República proponen gasto social diferenciado por regiones, según grado de pobreza, y el establecimiento de fondos regionales para la inversión territorial, especialmente en infraestructura y capital humano (Barón y Meisel, 2003; Bonet y Meisel, 2007; Galvis y Hahn, 2016). Los mecanismos de política pública que se aproximan, aunque lentamente, a estas recomendaciones son el sistema de transferencias condicionadas (post reformas 2001 y 2007) y no condicionadas 
(a partir del Sistema General de Regalías en 2012).

La presencia de clusters de regiones con efecto vecindario generalmente es positiva cuando incluyen un área metropolitana dinámica y, negativa, si son zonas periféricas, genera otro punto focal en estos estudios, a saber, la aplicación de políticas diferenciadas según zonas geográficas (Galvis y Meisel, 2013; Loaiza y Moncada, 2013). Un primer paso para la construcción de una política regional es la adecuada identificación de los fenómenos de aglomeración económica y derramamiento espacial, la cual plantea el reto de la medición que subsecuentemente se traslada al ámbito de la implementación. Así, la definición de áreas FUR en Colombia y del sistema de ciudades ha sido realizada por la OECD, el DNP y el Banco Mundial (DNP, 2014b; Sánchez-Serra, 2016). En particular, el sistema de ciudades se incorpora en la propuesta de la política general para el ordenamiento territorial del Plan Nacional de Desarrollo 2014-2018, donde se consignan principios de diferenciación para las regiones más pobres, e instrumentos de coordinación para la regulación e inversión territorial (DNP, $2014 a)^{10}$.

Finalmente, ante la divergencia en ingresos, pero convergencia en estándares de vida, autores como Royuela y García (2015) recomiendan la concentración de la producción (polarización), debido a la eficiencia en la escala de producción y mayor inversión social a nivel local, la cual traería el desarrollo de instituciones locales fuertes (pp. 235-236). Esta posición resulta más consecuente con las contribuciones de la NGE que explican la inevitabilidad de la aglomeración económica, con lo cual aspirar a la igualación en el ingreso per cápita de todas las regiones es irreal. Sin embargo, una excesiva aglomeración también puede afectar negativamente el crecimiento económico debido a

10 Aunque no todos estos elementos fueron sancionados en la Ley 1753 del 2015 (Congreso de la República, 2015), algunos sí permanecieron, complementándose con los Programas de Desarrollo con Enfoque Territorial - PDET (Decreto 893 de 2017 -MinAgricultura, 2017-). las deseconomías de escala y la congestión. En esta línea, se advierten estudios que muestran la diversidad de patrones de aglomeración, y no solamente el de megaciudades, que también generan eficiencia en la asignación de los recursos y la producción (Cuadrado-Roura y Aroca, 2013). Otro riesgo que entraña la vía de la polarización productiva está en la interdependencia entre equidad y crecimiento: brechas regionales en ingresos muy grandes pueden restringir el crecimiento económico como ha sido explicado por la literatura macroeconómica (Ros, 2013). En consecuencia, cerrar las brechas regionales en estándares de calidad de vida, a través de la inversión social, también depende de menores brechas en ingreso.

\section{Conclusiones}

Tres décadas de estudios sobre la convergencia regional, bajo el marco neoclásico tradicional, indican un agotamiento teórico y metodológico que motiva la incorporación más audaz, tanto de sus extensiones (crecimiento endógeno y NGE) como de otras influencias teóricas (enfoque de la demanda Keynesiano o Kaleckiano, el análisis institucional). Es central incorporar los arreglos institucionales que están en la base de la productividad y la competitividad, moldeados por la economía política y los problemas de agencia. Igualmente importante es reconocer los efectos de la economía global sobre las dinámicas regionales subnacionales que operan a través del comercio exterior y otros vínculos transnacionales.

La econometría sigue siendo una herramienta útil, en especial los modelos espaciales que han sido insuficientemente explorados y fundamentados teóricamente. Además, el uso de otros enfoques metodológicos, como los cualitativos (ej. estudios de caso) o mixtos, que identifiquen los mecanismos de la difusión tecnológica y la creación de ventajas competitivas localizadas, pueden aportar poder explicativo. La existencia de múltiples regímenes de crecimiento espacial y temporalmente localizados, en lugar de un único régimen válido en todo tiempo y lugar, es un fenómeno complejo que reclama una mirada teórica amplia y una aplicación flexible de métodos. 
La construcción de una política de desarrollo regional en Colombia encuentra una base valiosa en los estudios sobre convergencia regional, que confirma la divergencia y polarización de las últimas tres décadas, y la necesidad de focalización ante la inexistencia de mecanismos de nivelación socioeconómica automáticos. No obstante, esta construcción demanda más elaboración y debate público para responder preguntas como ¿se privilegia el crecimiento económico regional o el desarrollo socio-económico de las regiones? Así mismo, para ponderar explícitamente cuáles de los componentes de la política son neutrales al es- pacio y cuáles son específicos a este. Dentro de los primeros están las políticas generales de desarrollo, como las redistributivas de reducción de la pobreza, o las políticas nacionales de inversión en infraestructura, entre otras; dentro de los segundos están las políticas focalizadas que buscan mejorar las condiciones sociales y económicas de las regiones en crisis o más deprimidas. Establecer la complementariedad o sustituibilidad de estos dos componentes y las condiciones necesarias para su puesta en marcha, en una perspectiva de mediano y largo plazo, es un punto esencial para el desarrollo regional colombiano del siglo XXI.

\section{Referencias}

Acevedo, S. (2003). Convergencia y crecimiento económico en Colombia 1980-2000. Ecos de Economía, $7(17), 51-78$.

Aghion, P. y Howitt, P. (1998). Endogenous Growth Theory. MIT Press.

Aguirre, K. (2005). Convergencia en indicadores sociales en Colombia. Una aproximación desde los enfoques tradicional y no paramétrico. Revista Desarrollo y Sociedad, 56(2000), 147-176. https://doi. org/10.13043/DYS.56.5

Anselin, L. (1988). Spatial Econometrics: Methods and Models. Kluwer Academic.

Anselin, L. (1995). Local indicators of spatial association-LISA. Geographical Analysis, 27(2), 93-115. https:// doi.org/10.1111/j.1538-4632.1995.tb00338.x

Anselin, L. y Bera, A. (1998). Spatial dependence in linear regression models. En A. Ullhah y D. Giles (Eds.), Handbook of Applied Economic Statistics (pp. 237-289). Marcel Dekker.

Ardila, L. (2004). Gasto público y convergencia regional en Colombia. Revista Ensayos sobre política económica, (45), 222-268.

Arellano, M. (1988). An alternative transformation for fixed models with predetermined variables (Applied Economics Discussion Paper n 57). Oxford University. https://doi.org/10068/649426

Arellano, M. y Bond, S. (1991). Some test specification for panel data: Monte Carlo evidence and an application to employment equations. Review of Economic Studies, 58(2), 577-597. https://doi.org/1 0.2307/2297968

Arellano, M. y Bover, O. (1995). Another look at the instrumental variable estimation of error-components models. Journal of Econometrics, 68(1), 29-51. https://doi.org/10.1016/0304-4076(94)01642-D

Badinger, H., Müller, W. y Tondl, G. (2004). Regional Convergence in the European Union, 19851999: A Spatial Dynamic Panel Analysis. Regional Studies, 38(3), 241-253. https://doi. org/10.1080/003434042000211105

Barón, J. (2004). ¿Qué sucedió con las disparidades económicas regionales en Colombia entre 1980 y 2000? En A. Meisel (Ed.), Macroeconomía y regiones en Colombia (pp. 288-325). Banco de la República.

Barón, J. y Meisel, A. (2003). La descentralización y las disparidades económicas regionales en Colombia en la década de los noventa. Coyuntura Económica, XXXIII(2), 105-141. https://doi.org/10.32468/dtseru.36 
Barro, R. (1989). A Cross-Country Study of Growth, Saving, and Government (Working Paper n 2855). NBER. https://doi.org/10.3386/w2855

Barro, R. y Sala-i-Martin, X. (1991). Convergence across States and Regions. Brookings Papers on Economic Activity, 22(1), 107-182. http://doi.org/10.2307/2534639

Barro, R. y Sala-i-Martin, X. (1992a). Convergence. The Journal of Political Economy, 100(2), 223-252. https:// doi.org/10.1086/261816

Barro, R. y Sala-i-Martin, X. (1992b). Regional Growth and Migration (Working Paper $n^{\circ}$ 4038). NBER. https:// doi.org/10.3386/w4038

Barro, R. y Sala-i-Martin, X. (2003). Economic Growth. MIT Press.

Baumol, W. J. (1986). Productivity Growth, Convergence, and Welfare: What the Long-Run Data Show. The American Economic Review, 76(5), 1072-1085. https://doi.org/10.2307/1816469

Becker, G. S., Philipson, T. J. y Soares, R. R. (2005). The Quantity and Quality of Life and the Evolution of World Inequality. The American Economic Review, 95(1), 277-291. https://doi.org/10.1257/0002828053828563

Bernard, A. B. y Durlauf, S. N. (1995). Convergence in international output. Journal of Applied Econometrics, 10(2), 97-108. https://doi.org/10.1002/jae.3950100202

Birchenall, J. y Murcia, G. (1997). Convergencia regional: una revisión del caso colombiano. Desarrollo y Sociedad, (40), 273-308. https://doi.org/10.13043/dys.40.6

Blundell, R. y Bond, S. (1998). Initial conditions and moment restrictions in dynamic panel data models. Journal of Econometrics, 87(1), 115-143. https://doi.org/10.1016/S0304-4076(98)00009-8

Bonet, J. y Meisel, A. (2001). La convergencia regional en Colombia: una visión de largo plazo, 1926-1995. En A. Meisel (Ed.), Regiones, ciudades y crecimiento económico en Colombia (pp. 11-56). Banco de la República.

Bonet, J. y Meisel, A. (2007). Polarización del ingreso per cápita departamental en Colombia, 1975-2000. Ensayos sobre política económica, 25(54), 12-43.

Branisa, B. y Cardozo, A. (2009a). Regional Growth Convergence in Colombia Using Social Indicators (Discussion paper $n^{\circ} 195$ ). Ibero-America Institute for Economic Research.

Branisa, B. y Cardozo, A. (2009b). Revisiting the Regional Growth Convergence Debate in Colombia Using Income indicators (Discussion paper $\mathrm{n}^{\circ}$ 194). Ibero-America Institute for Economic Research.

Cárdenas, M. y Pontón, A. (1995). Growth and convergence in Colombia: 1950-1990. Journal of Development Economics, 47(1), 5-37. https://doi.org/10.1016/0304-3878(95)00003-8

Cardenas, M., Pontón, A. y Trujillo, J. P. (1993). Convergencia y migraciones inter-departamentales en Colombia: 1950-1989. Coyuntura Económica, 23(1), 111 -137. https://doi.org/10.2147/VHRM.S39726

Congreso de la República. (2015). Ley 1753. Por la cual se expide el Plan Nacional de Desarrollo 2014-2018. https://colaboracion.dnp.gov.co/CDT/Normograma/Ley\%201753\%20de\%202015.pdf

Crafts, N. (2000). Globalization and Growth in The Twentieth Century (Working Paper n 44). IMF

Cuadrado-Roura, J. R. y Aroca, P. (2013). Facing the Need for Regional Policies in Latin America. En J. R. Cuadrado-Roura y P. Aroca (Ed.), Regional Problems and Policies in Latin America. Advances in Spatial Science (pp. 21-42). Springer. https://doi.org/10.1007/978-3-642-39674-8_2

DNP -Departamento Nacional de Planeación-. (2014a). Bases para la formulación de la Política General de Ordenamiento Territorial. Texto para discusión. Insumo para el Plan Nacional de Desarrollo 2014-2018. DNP. 
DNP -Departamento Nacional de Planeación-. (2014b). Misión Sistema de Ciudades. Una política nacional para el sistema de ciudades colombiano con una visión de largo plazo. DNP. https://www.dnp.gov.co/ programas/vivienda-agua-y-desarrollo-urbano/desarrollo-urbano/Paginas/sistema-de-ciudades---libro. asp $x$

Elhorst, J. P. (2003). Specification and estimation of spatial panel data models. International Regional Science Review, 26(3), 244-268. https://doi.org/10.1177/0160017603253791

Fingleton, B. y López-Bazo, E. (2006). Empirical growth models with spatial effects. Papers in Regional Science, 85(2), 177-198. https://doi.org/10.1111/j.1435-5957.2006.00074.x

Fischer, M. M. y Getis, A. (2010). Handbook of Applied Spatial Analysis: Software Tools, Methods and Applications. Springer.

Fischer, M. M. y Stumpner, P. (2010). Income Distribution Dynamics and Cross-Region Convergence in Europe. En M. M. Fischer y A. Getis (Eds.), Handbook of Applied Spatial Analysis: Software Tools, Methods and Applications (pp. 599-628). Springer.

Franco, L. y Raymond, J. (2009). Convergencia económica regional: El caso de los departamentos colombianos. Ecos de Economía, 13(28), 167-197. https://doi.org/10.1344/105.000001675

Fujita, M., Krugman, P. y Venables, A. (1999). The Spatial Economy: Cities, Regions and International Trade. Cambridge.

Galvis, L. y Hahn, L. (2016). Crecimiento municipal en Colombia: el papel de las externalidades espaciales, el capital humano y el capital físico. Sociedad y Economía, (31), 149-174. https://doi.org/10.25100/sye. v0i31.3892

Galvis, L. y Meisel, A. (2001). El crecimiento de las ciudades colombianas y sus determinantes, 1973-1998. En A. Meisel (Ed.), Regiones, ciudades y crecimiento económico en Colombia (pp. 57-90). Banco de la República.

Galvis, L. y Meisel, A. (2013). Regional Inequalities and Regional Policies in Colombia: The Experience of the Last Two Decades. En J. R. Cuadrado-Roura y P. Aroca (Eds.), Regional Problems and Policies in Latin America. Advances in Spatial Science (pp. 197-223). Springer.

Galvis, L., Galvis, W. y Hahn-De-Castro, L. (2017). Una revisión de los estudios de convergencia regional en Colombia (Documentos de Trabajo sobre Economía Regional y Urbana ${ }^{\circ} 264$ ). Banco de la República.

Hahn-De-Castro, L. y Meisel, A. (2018). La desigualdad económica entre las regiones de Colombia, 1926 2016 (Cuadernos de historia económica n 47). Banco de la República. https://doi.org/10.32468/chee.47

Hsiao, C. (1986). Analysis of Panel Data. Cambridge University Press. https://doi.org/10.1017/ СвО9781139839327

Kenny, C. (2005). Why are we worried about income? Nearly everything that matters is converging. World Development, 33(1), 1-19. https://doi.org/10.1016/j.worlddev.2004.06.016

LeSage, J. P. y Pace, R. K. (2009). Introduction to Spatial Econometrics. Taylor \& Francis Group.

Loaiza, O. y Moncada, J. (2013). Un estudio sobre las disparidades regionales en Colombia a través del análisis exploratorio y confirmatorio de datos espaciales: 1985-2010 (Paper No. 47735). MPRA.

Lucas, R. (1988). On the mechanics of economic development. Journal of Monetary Economics, 22(1), 3-42. https://doi.org/10.1016/0304-3932(88)90168-7

Magrini, S. (2004). Regional (di)convergence. Handbook of Regional and Urban Economics, 4, 2741-2796. https://doi.org/10.1016/S1574-0080(04)80019-1

Martin, R. y Sunley, P. (1998). Slow Convergence? The New Endogenous Growth Theory and Regional Development. Economic Geography, 74(3), 201-227. https://doi.org/10.2307/144374 
Martínez, A. (2006). Determinantes del PIB per cápita de los departamentos colombianos 1975-2003. Archivos de Economía, 318, 1-28.

Meisel, A. (1993). ¿Polarización o convergencia? A propósito de Cárdenas, Pontón y Trujillo. Coyuntura Económica, 23(2), 153-161. http://hdl.handle.net/11445/2277

Meisel, A. y Galvis, L. (2009). Persistencia de las desigualdades regionales en Colombia: un análisis espacial. Revista del Banco De La República, 82(986), 37-70. https://doi.org/10.32468/dtseru.120

MinAgricultura -Ministerio de Agricultura y Desarrollo Rural-. (2017). Decreto 893 de 2017 "Por el cual se crean los Programas de Desarrollo con Enfoque Territorial PDET". https://www.funcionpublica.gov.co/ eva/gestornormativo/norma.php?i=81856

Quah, D. (1993). Galton's Fallacy and Test of the Convergence Hypothesis. The Scandinavian Journal of Economics, 95(4), 427-443. https://doi.org/10.2307/3440905

Quah, D. (1996a). Empirics for Economic Growth and Convergence. European Economic Review, 40(6), 1353 1375. https://doi.org/10.1016/0014-2921(95)00051-8

Quah, D. (1996b). Regional convergence clusters across Europe. European Economic Review, 40(3-5), 951 958. https://doi.org/10.1016/0014-2921(95)00105-0

Quah, D. (1997). Empirics for Growth and Convergence: Stratification, Polarization, and Convergence Clubs. Journal of Economic Growth, 2(1), 27-59. https://doi.org/10.1023/A:1009781613339

Rey, S. J. y Janikas, M. V. (2005). Regional convergence, inequality, and space. Journal of Economic Geography, 5(2), 155-176. https://doi.org/10.1093/jnlecg/lbh044

Rhenals, R., González, A. y Castaño, E. (1998). Crecimiento y Convergencia Regional en Colombia: El caso de Antioquia. Corporación para el Desarrollo de la Investigación y la Docencia Económica (CIDE).

Roberts, M. y Setterfield, M. (2010). Endogenous regional growth: a critical survey. En M. Setterfield (Ed.), Handbook of Alternatives Theories of Economic Growth (pp. 431-450). Edward Elgar Publishing Limited. https://doi.org/10.4337/9781849805582.00032

Rocha, R. y Vivas, A. (1998). Crecimiento Regional en Colombia: ¿Persiste la desigualdad? Revista de Economía del Rosario, 1(1), 67-108.

Romer, P. M. (1986). Increasing returns and long-run growth. Journal of Political Economy, 94(5), 1002-1037. https://doi.org/10.1086/261420

Ros, J. (2013). Rethinking Economic Development, Growth, and Institutions. Oxford University Press.

Royuela, V. y García, G. A. (2015). Economic and Social Convergence in Colombia. Regional Studies, 49(2), 219-239. https://doi.org/10.1080/00343404.2012.762086

Sánchez-Serra, D. (2016). Functional Urban Areas in Colombia (OECD Regional Development Working Papers $n^{\circ}$ 08). OECD Publishing. https://doi.org/10.1787/5jln4pn1 zqq5-en

Solow, R. (1956). A contribution to the theory of economic growth. The Quarterly Journal of Economics, 7O(5), 65-94. https://doi.org/10.2307/1884513

Soto, J. (1998). Crecimiento y convergencia departamental. Una aproximación de panel al caso colombiano 1960-1995 (tesis de maestria). Universidad de los Andes, Bogotá, Colombia.

Swan, T. W. (1956). Economic growth and capital accumulation. Economic Record, 32(63), 334-361. https:// doi.org/10.1111/j.1475-4932.1956.tb00434.x 